\title{
ON THE DISTRIBUTION OF ZEROS OF ENTIRE FUNCTIONS
}

\author{
A. R. REDDY
}

ABSTRACT. Let $f(z)$ be any transcendental entire function. Let $r_{k}$ denote the absolute value of the zero $z_{k}$ of $f^{(k)}(z)$ which is nearest to the origin. Alander, Erdös and Rényi, and Pólya have investigated the relation between $r_{k}$ and the growth of the function $f(z)$. Let $s_{k}$ denote the largest disk about the origin where $f^{(k)}(z)$ is univalent. Boas, Levinson, and Pólya have obtained some relations between the growth of the function $f(z)$ and $s_{k}$. Recently Shah and Trimble have sharpened the results of Boas and Pólya. We present here results in a different direction, generalizing the above quoted results. We also present results connecting the zero-free disks and the univalent disks about the origin of the normalized remainders of $f(z)$ with the growth of $f(z)$.

1. Introduction. There is extensive literature on the existence of zero-free disks for a sequence of derivatives of an entire function, as well as on disks of univalence for derivatives. Recently it has become clear that there are analogous results for a class of operators much more general than the operator of differentiation, the so-called D-operators (cf. [6]). These are defined as follows.

Let $\left\{d_{p}\right\}_{p=1}^{\infty}$ denote a nondecreasing sequence of positive numbers and let the operator $D$ transform the function $f(z)=\sum_{j=0}^{\infty} a_{j} z^{j}$ into $D f(z)=$ $\sum_{j=0}^{\infty} d_{j+1} a_{j+1} z^{j}$. In general for $k=0,1,2, \cdots$,

$$
D^{k} f(z)^{1}=\sum_{j=0}^{\infty} \frac{e_{j}}{e_{k+1}} a_{k+j} z^{j} \text {, where } e_{0}=1 \text { and } e_{j}=\left(d_{1} d_{2} \ldots d_{j}\right)^{-1} \text {. }
$$

For $d_{n}=n, D$ is the ordinary derivative; for $d_{n}=1, D$ is the shift operator $\sigma$, whose iterates are the nomalized remainders of the power series of the function; much less has been known about zero-free disks for $\sigma^{k} f(z)$ than for

Received by the editors July 5, 1973.

AMS (MOS) subject classifications (1970). Primary 30 A64, 30 A66, 30A36; Secondary $30 \mathrm{~A} 98,30 \mathrm{~A} 68$.

Key words and phrases. Zeros of entire functions, zero-free disks, univalent disks.

${ }^{1}$ We restrict that $\lim \sup _{n \rightarrow \infty}\left|a_{n} / e_{n}\right|^{1 / n}<\infty$; this restriction assures that each of $f, D f, \cdots, D^{k} f$, is entire (cf. $\left.[6, \mathrm{p} \cdot 350]\right)$. 
$f^{(k)}(z)$ (cf. [7]). By studying D-operators in general we not only show that many known results for derivatives appear as special cases of a general theory, but we also obtain the unexpected result that for the $D$-operators corresponding to certain sequences $\left\{d_{p}\right\}$ the functions $D^{k} f(z)$ have, so to speak, much scarcer zeros than is the case for $f^{(k)}(z)$. For example, if the lower order of $f$ is less than $a$ and $d_{k}=k^{1 / a}(\alpha>0)$, then Theorem 2 of this paper asserts that anywhere in the plane for every disk of arbitrarily large radius there exist infinitely many $k$ where $D^{k} f(z) \neq 0$.

Throughout our work we restrict $d_{j}$ by

$$
d_{j+k} \leq d_{k+1} d_{j} \text { where } k, j=1,2,3,4, \cdots
$$

\section{Lemmas.}

Lemma $1[2, \mathrm{p} .82]$. Let $f(z)=\sum_{j=0}^{\infty} a_{j} z^{j}$ be any entire function. Then for an infinity of $k$ and $j=0,1,2,3, \cdots$, we have $\left|a_{k+j}\right| \leq\left|a_{k}\right|\left|a_{k}\right|^{j / k}$ 。

Lemma 2 [9, (39)]。 Let $f(z)$ be an arbitrary entire function, $M(r)=$ $\max |z|=r f(z)$, and let $x=H(Y)$ denote the inverse function of $Y=\log M(x)$, then $\left|a_{n}\right|^{1 / n} H(n) \leq e(n=1,2,3, \cdots)$.

Lemma 3 [4, p。13]. Let $f(z)=\sum_{j=0}^{\infty} a_{j} z^{j}$ be any entire function of lower order $\lambda(0 \leq \lambda \leq \infty)$ and let $v(r)$ denote the central index, then

$$
\lim _{r \rightarrow \infty} \inf \frac{\log v(r)}{\log r}=\lambda .
$$

Lemma 4 [14, p. 24]. Let $f(z)=\sum_{j=0}^{\infty} a_{j} z^{j}$ be any entire function of or$\operatorname{der} \rho(0<\rho<\infty)$, lower order $\lambda(0 \leq \lambda \leq \rho<\infty)$, lower type $t$ and type $T$ $(0 \leq t \leq T \leq \infty)$, then

$$
\begin{aligned}
\lim _{r \rightarrow \infty} \inf \frac{v(r)}{r} & \leq \lambda T, \\
& \leq \rho t .
\end{aligned}
$$

Lemma 5. Let $f(z)$ be any entire function. Then $D^{k-1} f(z)$ is univalent in a disk of radius $R$ if

$$
\sum_{j=1}^{\infty}(j+1) \frac{\left|a_{k+j}\right|}{\left|a_{k}\right|} \frac{e_{j+1} e_{k} R^{j}}{e_{j+k} e_{1}}<1 .
$$

Proof. It is clear from the definition that for $Z_{1} \neq Z_{2}$ and $\left|Z_{1}\right|<R,\left|Z_{2}\right|<R$,

$$
\begin{aligned}
\left|\frac{D^{k-1} f\left(z_{1}\right)-D^{k-1} f\left(z_{2}\right)}{z_{1}-z_{2}}\right| & =\left|\sum_{j=1}^{\infty} \frac{e_{j}}{e_{k+j-1}} a_{k+j-1} \frac{\left(z_{1}^{j}-z_{2}^{j}\right)}{\left(z_{1}-z_{2}\right)}\right| \\
& \geq \frac{\left|a_{k}\right| e_{1}}{e_{k}}-\sum_{j=2}^{\infty} j R^{j-1} \frac{\left|a_{k+j-1}\right| e_{j}}{e_{j+k-1}} .
\end{aligned}
$$


If $\left|a_{k}\right| e_{1} / e_{k}>\sum_{j=2}^{\infty} j R^{j-1}\left|a_{j+k-1}\right| e_{j} / e_{j+k-1}$, then clearly $D^{k-1} f(z)$ is univalent for $|Z|<R$, hence the lemma.

3. Zero-free disks for $D^{k} f(z)$.

Theorem 1. Let $f(z)=\sum_{j=0}^{\infty} a_{j} z^{j}$ be an entire function, and let $\psi_{k}$ denote the absolute value of the zero $Z_{k}$ of $D^{k} f(z)$ which is nearest to the origin, then denoting by $x=H(Y)$ the inverse function of $Y=\log M(x)$, and with the assumption (1), we have

$$
\limsup _{k \rightarrow \infty} \frac{d_{k+1} \psi_{k}}{H(K)} \geq \frac{1}{2 e} .
$$

Proof. From the definition of $D^{k} f(z)$ along with Lemma 1, we have, for all those values of $k$ for which Lemma 1 is valid,

$$
\begin{aligned}
\left|\frac{D^{k} f(z)}{D^{k} f(0)}-1\right| & \leq \sum_{j=1}^{\infty} \frac{e_{k} e_{j}}{e_{k+j}} \frac{\left|a_{k+j}\right|}{\left|a_{k}\right|}|z|^{j} \\
& \leq \sum_{j=1}^{\infty} \frac{d_{k+1} d_{k+2} \cdots d_{k+j}}{d_{1} d_{2} \cdots d_{j}}\left|a_{k}\right|^{j / k}|z|^{j} \\
& \leq \sum_{j=1}^{\infty} d_{k+1}^{j}\left|a_{k}\right|^{j / k} R^{j}=\frac{1}{1-\left|a_{k}\right|^{1 / k} d_{k+1} R}-1
\end{aligned}
$$

by choosing $\left|a_{k}\right|^{1 / k} d_{k+1} R<1$.

Now it is clear from (4) that if $1 /\left(1-\left|a_{k}\right|^{1 / k} d_{k+1} R\right)-1<1$ then $D^{k} f(z)$ $\neq 0$ for $|z| \leq R$.

In other words if $R<1 / 2\left|a_{k}\right|^{1 / k} d_{k+1}$, then $D^{k} f(z) \neq 0$ for $|z| \leq R$. That is

$$
\psi_{k} \geq \frac{1}{2\left|a_{k}\right|^{1 / k_{d}}{ }_{k+1}} .
$$

Now the required result (1) follows from (5) and Lemma 2.

Remarks. We have from (2) for any entire function $\lim \sup _{k \rightarrow \infty} d_{k+1} \psi_{k}=\infty$. For $d_{k}=k$, we have the result of Erdös and Rényi $[9,(47)]$ as a particular case.

Corollary 1. Let $C=C_{k}(f)$ denote the absolute value of the zero $\left|z_{k}\right|$ of $\sigma^{k} f(z)$ (kth normalized remainder), which is closest to the origin, then for any entire function $\lim \sup _{k \rightarrow \infty} C_{k}=\infty$.

Proof. This follows easily from (2) by choosing $d_{k}=1$.

Theorem 2. Let $f(z)=\sum_{j=0}^{\infty} a_{j} z^{j}$ be an entire function of lower order 
$\lambda(0 \leq \lambda \leq \infty)$. If $\psi_{k}$ is defined as in Theorem 1 , then

$$
\limsup _{k \rightarrow \infty} \frac{\log \psi_{k} d_{k+1}}{\log _{k}} \geq \frac{1}{\lambda} \text {. }
$$

Proof. As usual

$$
\left|\frac{D^{k} f(z)}{D^{k} f(0)}-1\right| \leq \sum_{j=1}^{\infty} \frac{e_{k} e_{j}}{e_{k+j}} \frac{\left|a_{k+j}\right|}{\left|a_{k}\right|}|z|^{j} .
$$

If $f(z)$ is entire, then the radius of convergence $C=\infty$, that is, $\left|a_{n}\right|^{1 / n} \rightarrow 0$, and thus one can find for any $B>0(B<C)$ an infinity of values of $k$ for which $\left|a_{k+j}\right|<\left|a_{k}\right| B^{-j}(j=1,2,3, \cdots)$. Now by substituting this in the above inequality, we have

$$
\left|\frac{D^{k} f(z)}{D^{k} f(0)}-1\right| \leq \sum_{j=1}^{\infty} \frac{e_{k} e_{j}}{e_{k+j}} \frac{|z|^{j}}{B^{j}} \leq \sum_{j=1}^{\infty} d_{k+1}^{j}|z|^{j} B^{-j} .
$$

We choose here $d_{k+1}|z|<B$, so that we have from (6)

$$
\left|\frac{D^{k} f(z)}{D^{k} f(0)}-1\right| \leq \sum_{j=1}^{\infty} d_{k+1}^{j}|z|^{j} B^{-j}=\frac{1}{1-d_{k+1} R / B}-1 .
$$

As earlier it is clear, from (7), $D^{k} f(z) \neq 0$, for $|z| \leq R$ if $R<B / 2 d_{k+1}$, that is, $\psi_{k} \geq B / 2 d_{k+1}$.

Now we choose here $B=r, k=v(r)$ the central index of $f$, and clearly $C>r$. Then

$$
\frac{\log r}{\log v(r)} \leq \frac{\log d_{k+1} \psi_{k}}{\log v(r)}+\frac{\log 2}{\log v(r)}
$$

This inequality along with Lemma 3 gives us the required result.

Remarks. If $\lambda<a$ and $d_{k}=k^{1 / a}(\alpha>0)$, then from Theorem 2 we have $\lim \sup _{k \rightarrow \infty} \psi_{k}=\infty$. From this it is clear that, if $\alpha$ is very large, $\lambda$ can be very large, thus we get even for large $\lambda$, very large zero free disks tending to infinity. In fact lim $\sup _{k \rightarrow \infty} \psi_{k}=\infty$. implies that anywhere in the plane for every disk of arbitrarily large radius, there exist infinitely many $k$ where $D^{k} f(z) \neq$ 0 . On the other hand, in the work of $\AA^{\circ}$ lander [2], $r_{k} \rightarrow 0$ as $\rho>1$, and in Boas and Reddy [5], the radius of zero free disks tends to zero when $\rho>2$. For $d_{k}=k$, Theorem 2 improves the result of Alander [2, Theorem 2] and Pólya [13, p. 18]. This also improves another result of Pólya [12,Theorem II] replacing the restriction $\rho<2 / 3$ by $\lambda<2 / 3$. This suggests that Pólya's hypothetical Theorem A [13, p. 182] may be true for any order $\rho$ as long as the lower order $\lambda$ is less than $2 / 3$. 
Corollary 2. Let $f(z)=\sum_{j=0}^{\infty} a_{j} z^{j}$ be an entire function of lower order $\lambda(0 \leq \lambda \leq \infty) . C_{k}$ is defined as in Corollary 1 ; then

$$
\limsup _{k \rightarrow \infty} \frac{\log C_{k}}{\log _{k}} \geq \frac{1}{\lambda}
$$

Proof. This follows from Theorem 2 by taking $d_{k}=1$.

Theorem 3. Let $f(z)=\sum_{j=0}^{\infty} a_{j} z^{j}$ be an entire function of order $\rho(0<\rho$ $<\infty)$, lower order $\lambda \geq 0$, lower type $t \geq 0$, type $T \leq \infty$. Let $\psi_{k}$ denote the absolute value of the zero $Z_{k}$ of $D^{k} f(z)$ which is closest to the origin; then under hypothesis (1), we have

$$
\begin{aligned}
\limsup _{k \rightarrow \infty} \frac{d_{k+1} \psi_{k}}{k^{1 / \rho}} \geq \frac{1}{2(\lambda T)^{1 / \rho}}, & \geq \frac{1}{2(\rho t)^{1 / \rho}} .
\end{aligned}
$$

Proof. The proof of this theorem follows exactly on the same lines as that of Theorem 2, with one difference. We use Lemma 4 instead of Lemma 3; hence we omit the proof.

Corollary 3. Let $\lambda, \rho, t$ and $T$ have the same meaning as in Theorem 3. Then,

$$
\begin{aligned}
& \limsup _{k \rightarrow \infty} \frac{C_{k}}{k^{1 / \rho}} \geq\left[2(\lambda T)^{1 / \rho}\right]^{-1}, \\
& \geq\left[2(\rho t)^{1 / \rho}\right]^{-1} .
\end{aligned}
$$

This is a special case of Theorem 3 for $d_{k}=1$.

Theorem 4. Let $f(z)$ be an analytic function for $|z|<r$, not a polynomial. $\psi_{k}$ denotes the absolute value of the zero of $D^{k} f(z)$ which is nearest to the origin. Then under the assumption (1), we have

$$
\limsup _{k \rightarrow \infty} d_{k+1} \psi_{k} \geq \frac{r}{2} \text {. }
$$

Proof. If $f(z)$ is analytic for $|z| \leq r$, then $\lim \sup _{j \rightarrow \infty}\left|a_{j}\right|^{1 / j} \leq 1 / r$; from this we have for any $\epsilon>0$ that there exist infinitely many $k$ for which $\left|a_{k+j} / a_{k}\right|$ $\leq(1+\epsilon)^{j} r^{-j}, j=0,1,2, \cdots$. This along with (3) gives us

$$
\frac{\mid D^{k} f(z)}{D^{k} f(0)}-1 \mid \leq \sum_{j=1}^{\infty}\left\{\frac{d_{k+1}(1+\epsilon) R}{r}\right\}^{j}=\frac{1}{1-d_{k+1}(1+\epsilon) R / r}-1,
$$

because of the restriction that $d_{k+1}(1+\epsilon) R<r$. As earlier it is clear from this that

$$
D^{k} f(z) \neq 0 \text { for }|z| \leq R \text {, if } R<r / 2 d_{k+1}(1+\epsilon) \text {, }
$$


hence $\lim \sup _{k \rightarrow \infty} d_{k+1} \psi_{k} \geq r / 2, \epsilon$ being arbitrary.

Remarks: For $d_{k}=1$, there exist functions $f(z)$ with radius of convergence 1, for which lim inf ${ }_{k \rightarrow \infty} \psi_{k}$ can be determined precisely. It is known $\left[7,(1.2)\right.$ and Lemma 3] that $\lim \sup _{k \rightarrow \infty} \psi_{k}=\lim \sup _{k \rightarrow \infty} C_{k}(f)=1 / P$, where $1.7818<$ $P<1.82$. Given any positive small number $\beta$, it is possible to construct functions $f(z)$, which are analytic in the unit circle for which $\lim \inf \psi_{k} \leq \beta$.

For example, let $f(z)=1-\delta z+z^{2}-\delta z^{3}+z^{4}-\delta z^{5}+\cdots, \delta>0$; i.e.

$$
f(z)=(1-\delta z)\left(1+z^{2}+z^{4}+\cdots\right) .
$$

For this function, it is easy to verify that

Hence

$$
f(z)=\sigma^{2} f(z)=\sigma^{4} f(z)=\cdots=\sigma^{2 k} f(z), \text { for } k=0,1,2, \cdots
$$

$$
\sigma^{2 k_{f}}(z)=(1-\delta z)\left(1+z^{2}+z^{4}+\cdots\right), \quad \delta \geq 1 / \beta .
$$

From this it follows easily that $\lim _{\inf } \psi_{k \rightarrow \infty} \psi_{k}=1 / \delta \leq \beta$.

We would like to point out here that there exist entire functions $f(z)$ (cf. [10]) for which zeros of $D^{k} f(z)$ can be determined with precision.

\section{Disks of univalence.}

Theorem 5. Let $f(z)=\sum_{j=0}^{\infty} a_{j} z^{j}$ be any entire function. $U_{k}$ denotes the largest disc about the origin where $D^{k} f(z)$ is univalent $(k=1,2, \ldots)$. Then denoting by $x=H(Y)$ the inverse function of $Y=\log M(x)$, we have under hy. pothesis (1)

$$
\limsup _{k \rightarrow \infty} \frac{d_{k+1} U_{k-1}}{H(k)} \geq \frac{1}{4 e} \text {. }
$$

Proof. It is known from Lemma 5 that $D^{k-1} f(z)$ is univalent for $|z|<R$, if

$$
\sum_{j=1}^{\infty} 2^{j} \frac{e_{j} e_{k}}{e_{j+k}}\left|\frac{a_{j+k}}{a_{k}}\right| R^{j}<1 .
$$

It is easy to see that the right-hand side of (9) follows from the proof of Theorem 1, by replacing $R$ in (3) by $2 R$. Therefore all the calculations based on Theorem 1 are valid for (9) with $R$ replaced by $2 R$ in (4). Hence $D^{k-1} f(z)$ is univalent for $|z|<R$ if

$$
2 R<\frac{1}{2\left|a_{k}\right|^{1 / k} d_{k+1}} .
$$

Inequality (10) along with Lemma 2 gives us the required result. Similarly we can prove

Theorem 6. Let $f(z)=\Sigma_{j=0}^{\infty} a_{j} z^{j}$ be an entire function of lower order $\lambda \geq 0, U_{k}$ denotes the radius of the largest disk about the origin where 
$D^{k} f(z)$ is univalent. Then under assumption (1), we have

$$
\limsup _{k \rightarrow \infty} \frac{\log U_{k-1} d_{k+1}}{\log k} \geq \frac{1}{\lambda} .
$$

Theorem 7. Let $f(z)$ be an entire function of order $\rho(0<\rho<\infty)$, lower order $\lambda$, lower type $t$ and type $T(0 \leq t \leq T \leq \infty)$. $U_{k}$ denotes the radius of the largest disk about the origin where $D^{k} f(z)$ is univalent. Then under assumption (1), we have

$$
\underset{k \rightarrow \infty}{\limsup \frac{U_{k-1} d_{k+1}}{k^{1 / \rho}} \geq[4(\lambda T)]^{-1},} \geq
$$

Theorem 8. Let $f(z)$ be regular for $|z|<r$ and not a polynomial. $U_{k}$ denotes the radius of the largest disk about the origin where $D^{k} f(z)$ is univa. lent. Then under assumption (1),

$$
\limsup _{k \rightarrow \infty} d_{k+1} U_{k-1} \geq \frac{r}{4} \text {. }
$$

Remarks on Theorems 5 and 6 . For any entire function we have from Theorem 5, lim sup $\operatorname{sum}_{k \rightarrow+1} d_{k-1}=\infty$ 。 For $d_{k}=k$, this includes the result of Shah and Trimble as a special case. For the case $d_{k}=k$, Theorem $6 \mathrm{im}$ proves the result of Pólya [13, p. 181]; this includes also the result of Shah and Trimble $[15,(3.3)]$. For $d_{k}=k^{1 / a}\left(\alpha_{>}>0\right)$, one has from Theorem 6 for a sequence of values of $k, U_{k-1} \geq k^{(\lambda+\epsilon)^{-1}-a^{-1}}$, that is $U_{k-1} \uparrow \infty$ if $\lambda<\alpha$. In fact lim $\sup _{k \rightarrow \infty} U_{k}=\infty$ implies that for each large disk anywhere in the plane, there exist infinitely many $k$, for which $D^{k} f(z)$ is univalent. This result is stronger than the known results in this direction.

Let $V_{k}$ denote the largest disk about the origin, where $\sigma^{k} f(z)$ is univalent; then with the help of Theorems 5, 6 and 7, we can replace $C_{k}$ by $2 V_{k-1}$ in Corollaries 1, 2 and 3.

Theorems 5, 6, 7 and 8 can be extended easily to the $U_{q}$-radius studied in [8]; the details are left to the reader.

My thanks are due to Professor R. P. Boas for suggesting this investigation and also for his valuable suggestions in the preparation of this paper. I would like to thank the referee for his comments.

\section{BIBLIOGRAPHY}

1. M. Alander, Sur le déplacement des zéros des fonctions entières par leur dérivation, Thesis, Upsala, 1914.

2. - Sur les dérivées successives des fonctions régulières, Opuscula Mathematica, A. Wiman dedicata, Lund, 1930, 79-93. 
3. R. P. Boas, Univalent derivatives of entire functions, Duke Math. J. 6 (1940), 719-721. MR 2, 82 .

4. - Entire functions, Academic Press, New York, 1954. MR 16, 914.

5. R. P. Boas and A. R. Reddy, Zeros of successive derivatives of entire functions, Bull. Amer. Math. Soc. 79 (1973), 64-65; J. Math. Anal. Appl. 42 (1973), 466-473.

6. J. D. Buckholtz and J. L. Frank, Whittaker constants, Proc. London Math. Soc. (3) 23 ( 1971 1), 348-370. MR 45 \#5358.

7. J. D. Buckholtz and J. K. Shaw, Zeros of partial sums and remainders of power series, Trans. Amer. Math. Soc. 166 (1972), 269-284. MR 45 \#8810.

8. D. M. Campbell, Locally univalent functions with locally univalent derivatives, Trans. Amer. Math. Soc. 162 (1972), 395-409.

9. P. Erdös and A. Rényi, On the number of zeros of successive derivatives of analytic functions, Acta Math. Acad. Sci. Hungar. 7 (1956), 125-144. MR 18, 201.

10. G. H. Hardy, On the zeros of a class of integral functions, Messenger of Math. 34 (1905), 97-101.

11. N. Levinson, $A$ theorem of Boas, Duke Math. J. 8 (1941), 181-182. MR 2, 274 .

12. G. Pólya, Some problems connected with Fourier's work on transcendental equations, Quart. Math. 1 (1930), 622-634.

13. - On the zeros of derivatives of a function and its analytic character, Bull. Amer. Math. Soc. 49 (1943), 178-191. MR 4, 192.

14. A. R. Reddy, Note on a theorem of Erdös and Rényi, Acta Math. Acad.Sci. Hungar. 20 (1969), 241-243. MR 38 \#6067.

15. S. M. Shah and S. Y. Trimble, Univalent functions with univalent derivatives. II, Trans. Amer. Math. Soc. 144 (1969), 313-320. MR 40 \#2841.

DEPARTMENT OF MATHEMATICS, UNIVERSITY OF TOLEDO, TOLEDO OHIO 43606 